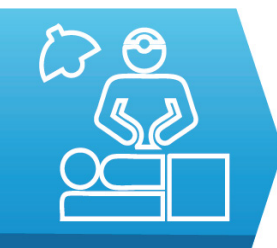

SURGERY

\title{
A rare case of idiopathic ileocolic intussusception in an adult
}

Muniba Mehmood ${ }^{1}$, Uzair Yaqoob², Khaled Abdullah Rage², Hina Khan², Mujeeb Rehman Abbasi ${ }^{2}$, Khursheed Ahmed Samo ${ }^{1}$

1) Dr. Ruth K. M. Pfau Civil Hospital, Karachi, Pakistan

2) Dow University of Health Sciences, Karachi, Pakistan

DOI: $10.15386 / \mathrm{mpr}-1847$

Manuscript received: 07.08.2020

Received in revised form: 25.11 .2020

Accepted: 13.12.2020

Address for correspondence:

ozair_91393@hotmail.com

This work is licensed under a Creative Commons Attribution-NonCommercialNoDerivatives 4.0 International License

\begin{abstract}
Intussusception is an invagination of one segment of the bowel into its adjoining segment. In children, the cause is usually benign, while in adults it is secondary to a mass lesion. Here we present a case in which the preoperative diagnosis of intussusception secondary to colonic mass was made, but no definitive cause was identified by histopathology. A 30-year-old male presented with abdominal pain, altered bowel habits, weight loss, loose motions, bleeding per rectum, and vomiting. The abdomen was distended, firm, mildly tender, and guarded. A vague mass of $15 \mathrm{x}$ $10 \mathrm{~cm}$ was palpated on the left upper quadrant. X-ray and ultrasound showed dilated small bowel. A computed tomography scan suggested ileoileal intussusception. Colonoscopy showed a growth at $15 \mathrm{~cm}$ of the anal verge. Exploratory laparotomy was performed, showing the presence of ileocolic intussusception with two large perforations. Subtotal colectomy with ileostomy was done. The histopathological examination showed signs of perforation.
\end{abstract}

Keyword: digestive system, surgical procedure, intestinal invagination, intussusception

\section{Introduction}

Intussusception, a medical condition in which one segment of the bowel loop invaginates into its adjoining segment, may occur in all age groups. Intussusception is more common in the pediatric population where the ratio of child to adult is around 20:1 [1,2]. In children, the cause is usually benign and they may present with a classic triad of abdominal pain, vomiting, and bleeding per rectum (PR) [3].

On the other hand, in adults, intussusception is merely secondary to pathologic conditions such as polyps, lipoma, malignancies, and Meckel's diverticulum [4]. Its etiology, clinical symptoms, diagnostic approach, and management differ from childhood intussusception. Adult intussusception accounts for around 5\% of all cases of intussusception and only $1-5 \%$ of intestinal obstructions in adults [5]. It is estimated that in adults around
2-3 cases $/ 1,000,000$ population/ year represent intussusception [6]. In adults, diagnosis of intussusception is challenging and it mimics many alternative diagnostics. It may lead to severe complications if not diagnosed properly $[7,8]$.

Adult intussusception is an uncommon disease. Here we present a case of intussusception in a 30-yearold patient, where the preoperative diagnosis of intussusception secondary to colonic mass (most likely malignant) was made based on history, examination, imaging, and endoscopic investigations, but no definitive cause was identified by histopathology. Although the management of adult intussusception is straightforward, in our case there was a huge length of a large bowel involved so extensive surgery was performed and a large portion of the bowel was resected. It is essential to consider a rare differential diagnosis while dealing with a 
patient's history, just like we focused on the presentation, diagnosis, and management of idiopathic adult ileocolic intussusception despite being a rare entity.

\section{Case presentation}

A 30-year-old male with no known comorbidities presented to the emergency department of Dr. Ruth Pfau Civil Hospital in April 2019 with complaints of abdominal pain, altered bowel habits, weight loss, and vomiting for the previous five months, plus diarrhea and bleeding PR for two days.

Although not documented, he had been to various clinics in his hometown, but there was no adequate relief. He had been in a usual state of health five months back when intermittent colicky abdominal pain started, first in the umbilical region, which then became generalized. The pain was associated with on and off vomiting, diarrhea, and constipation. The pain was relieved temporarily by taking painkillers. He also noticed some unintentional weight loss. For the last two days before arrival, symptoms got aggravated and he was having bleeding PR, two episodes per day.

On presentation, he was vitally stable, anemic, dehydrated, lying comfortably on the bed, well oriented to time, place, and person.

The abdomen was distended with no visible scars. On palpation, it was firm, mildly tender, and guarded on the periumbilical and left upper quadrant. A vague mass of around $15 \times 10 \mathrm{~cm}$ was also palpated on the left upper quadrant.

Based on history and examination findings, differential diagnosis was neoplasia, inflammatory bowel disease, diverticulosis, infective colitis, and angiodysplasia. The patient was kept nil per os and a nasogastric (NG) tube was passed. Intravenous fluids and antibiotics were also given.

Abdominal X-ray showed a dilated small bowel loop on the right side. Abdominal ultrasound abdomen showed multiple thick-walled bowel loops on the whole lower side, most likely due to inflammation. Plain computed tomography (CT) scan of the abdomen and pelvis showed telescoping of one intestinal segment into another making multiple hypo and hyperdense concentric rings in the left iliac fossa suggestive of ileoileal intussusception. The ileocecal junction and cecum were pulled medially and inferiorly. Mild free fluid was seen around the lesion in the left iliac fossa and left paracolic gutter (Figure 1). After the CT examination, a provisional diagnosis of large bowel obstruction secondary to colonic mass was made. Colonoscopy was performed on the $2^{\text {nd }}$ day of admission which showed a growth at $15 \mathrm{~cm}$ of the anal verge. The scope tube could not pass beyond the growth (Figure 2). Multiple biopsies were taken which showed inflammation on histopathology. No dysplasia, granuloma, or malignancy were seen.
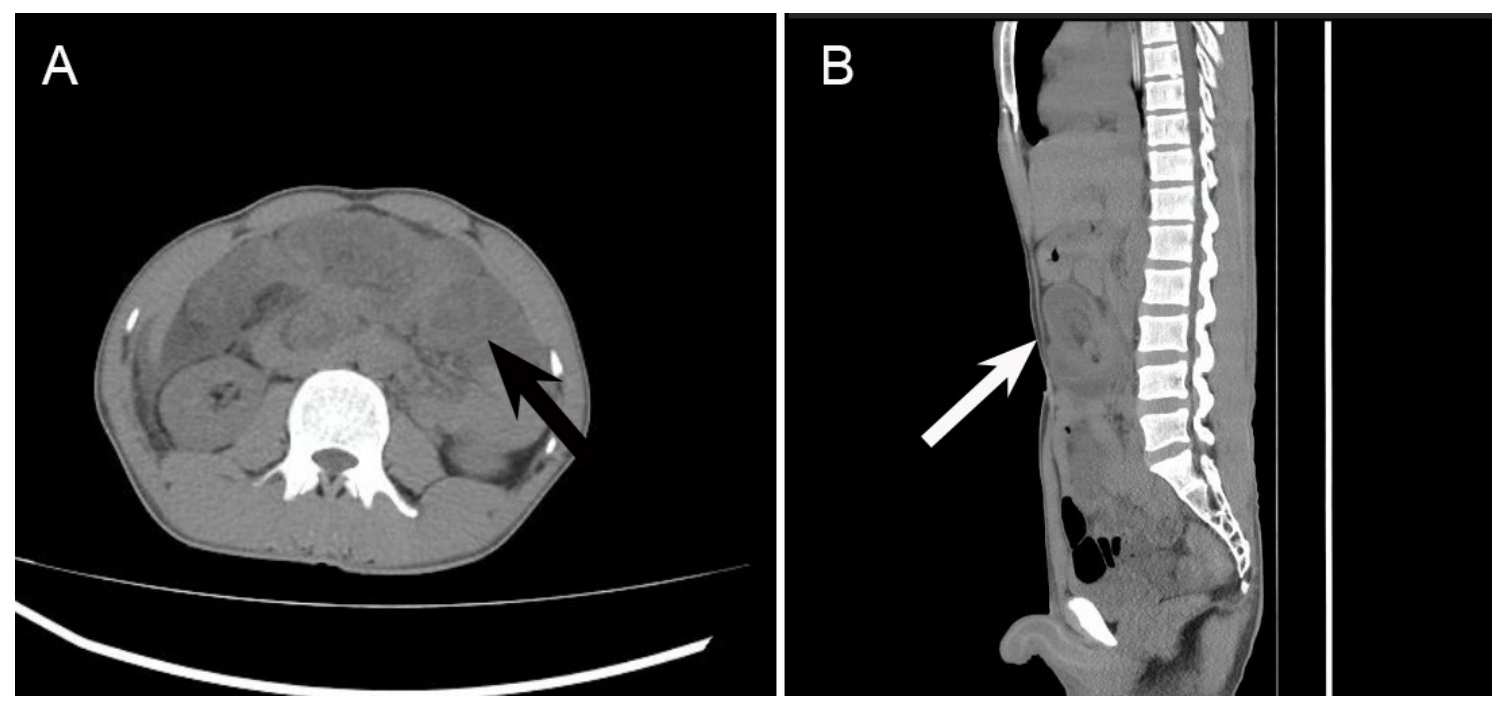

Figure 1. Computed tomography scan of the abdomen without contrast (A) Axial view showing hypo and hyperdense concentric rings (arrow) suggesting ileoileal intussusception; (B) Sagittal view showing a soft tissue sausage-shaped mass (arrow) suggestive of intestinal intussusception. 


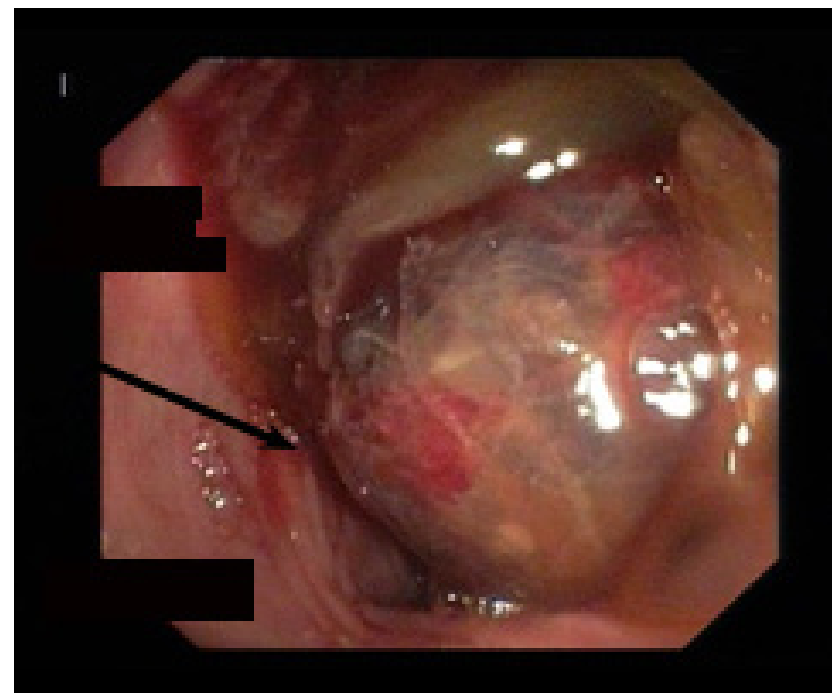

Figure 2. Colonoscopic view showing a suspected malignant growth found $15 \mathrm{~cm}$ from the anal verge (arrow).
Since a tumor mass was suspected as a cause of obstruction, we planned for a contrast CT scan and oncology review but on $3^{\text {rd }}$ day of admission, the patient's condition deteriorated. His NG output was increased with generalized abdominal tenderness and distension observed. A plan of exploratory laparotomy and proceed was made. Operative findings were ileocolic intussusception till the rectosigmoid junction with dilation of the proximal small bowel loops. The involved bowel was thickened and inflamed. Two large perforations were observed in the descending colon with $100 \mathrm{ml}$ of reactionary fluid and minimal contamination. No other pathology was found. Subtotal colectomy with end ileostomy at $45 \mathrm{~cm}$ away from ileocecal junction was made (Figure 3).
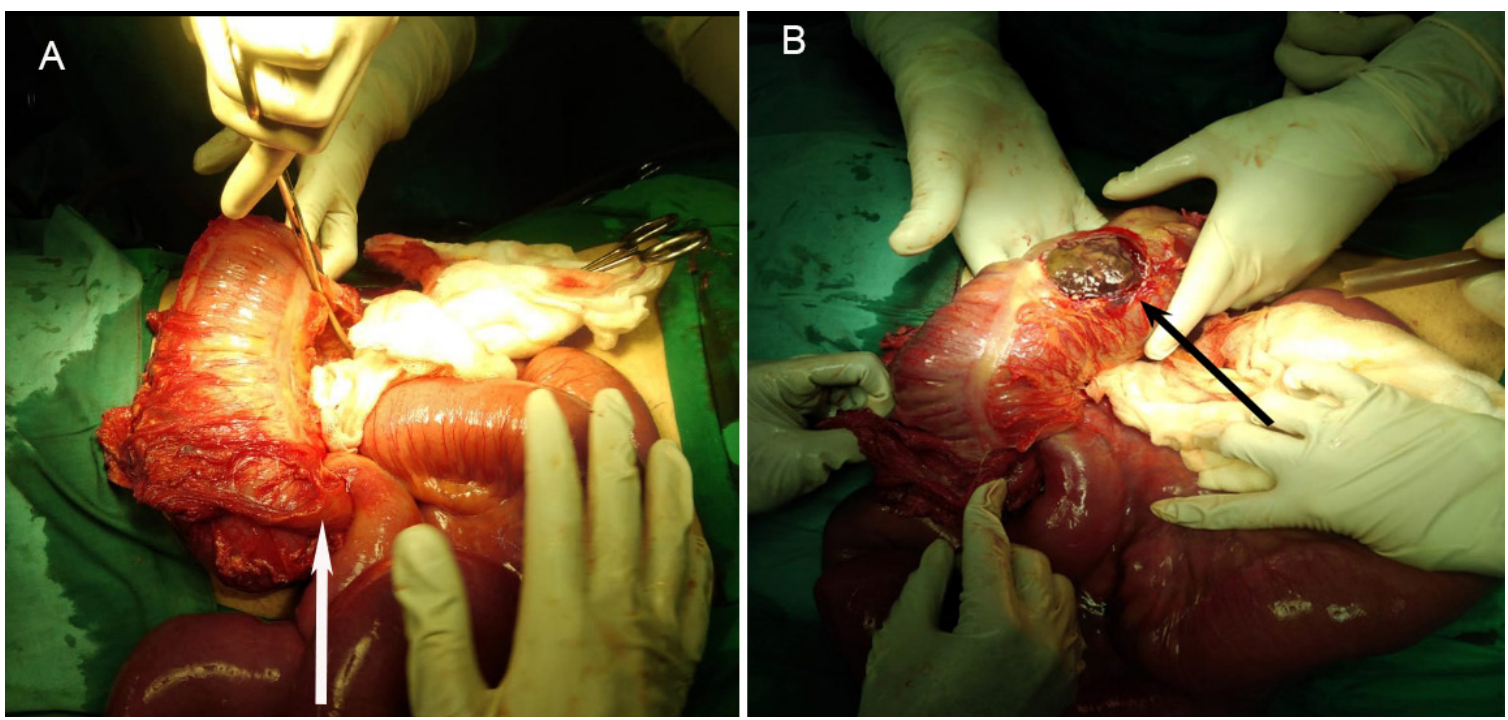

Figure 3. Intraoperative views showing (A) invagination of ileum with ileocecal junction into the colon (arrow) and (B) a $3 \times 3 \mathrm{~cm}$ perforation on the descending colon.

The patient was orally allowed feeding and the stoma started functioning on the second postoperative day. He was mobilized out of bed on day 3 of the operation and discharged on the $10^{\text {th }}$ day from admission.

When the patient was followed-up after a week, the histopathological examination of the intraoperative specimen showed extensive mucosal ulceration, transmural inflammation, and gangrenous necrosis compatible with perforation. No dysplasia, microorganisms, granuloma, or malignancy was seen; 29 lymph nodes were recovered that showed reactive changes only. Proximal resection margins were unremarkable. The patient was followed in OPD and the reversal of stoma was done by performing a laparotomy with hand-sewn ileocolic anastomosis. No complications occurred during the postoperative period. 


\section{Discussion}

Intussusception is mainly considered a disease of infancy and early childhood. Adult intussusception is easily differentiable from the pediatric one, where the cause is usually unknown $[2,9]$. According to the site, it is either ileal, ileocecal, colonic, ileocolic, or it may involve the whole of the intestine [10]. The ileocolic intussusception is defined as an invagination of the ileum through the ileocecal valve into the colon. It accounts for $15 \%$ of all intussusceptions. The normal anatomical position of the ileocecal valve and the appendix is usually preserved [11]. This finding of ileocolic intussusception is rare, as terminal ileum along with cecum slides into adjacent bowel till rectosigmoid junction, one loop of terminal ileum invaginated through the ileocecal valve into the cecum up to the rectosigmoid junction.

Small bowel tumors are rare, accounting for around $1-2 \%$ of all gastrointestinal tumors [12]. Benign tumors are still rarer and represent about $30 \%$ of all the small bowel tumors [13]. These are the second most common group of benign tumors after gastrointestinal stromal tumors [14]. One of the benign tumors i.e. intestinal lipomas are more common in females and occurs in the older population [15]. The terminal ileum is the most frequent site of involvement [13]. These are also the most common benign cause of colonic intussusception. Almost $90 \%$ of cases in adult intussusception are due to secondary pathology that set out as the underlying lead point. On the other hand, two-thirds of the colonic intussusceptions are reported to be associated with malignancy [16]. In our case, since the mass was initially considered colonic based on history and examination, a rectal tumor was kept in the differential diagnosis.

In adults with intussusception, symptoms are often non-specific and long-standing. The patient usually presents with abdominal pain, accounting for $71-90 \%$, with vomiting and bleeding PR as the next most common symptom [17]. Pain is usually colicky, periodic, and intermittent, causing difficulty in diagnosis with only half of the patients being diagnosed with intussusception before the surgery and abdominal mass found in $24-42 \%$ of cases [18]. The current case presented with similar symptoms.

Ultrasound examination can rule out an intussusception in approximately 86\%. Typically, ileocolic intussusception has an impression of a peripheral hypoechoic ring (the target sign) with central echogenicity (the pseudo kidney sign). These correspond to the bowel wall surrounding hypoechoic necrotic fat contained within the intussusception. Most commonly it is found on the right side with a diameter of 2-4 cm [19]. In our case, thick-walled bowel loops were found on the whole lower side of the abdomen and the impression of small bowel inflammation was given.

Due to the widespread use of CT scans, it has been reported to be the most useful imaging technique with an accuracy of $58-100 \%$ and specificity of $57-71 \%$ [2,20]. CT usually reveals a well-demarcated, smooth, sausage-shaped mass with two components (an inner intussusceptum and outer intussuscipiens) and eccentric fat representing the intussuscepted mesenteric fat [20]. On endoscopy, other characteristics are the "Cushing sign" and "Naked fat sign". In our case, a CT scan without contrast was done and the images were suggestive of intussusception. However, on colonoscopy, findings were highly in favor of a malignant tumor.

In children, the pneumatic or hydrostatic reduction is usually the treatment of choice. However, in adults, almost $70-90 \%$ of cases of intussusception require definitive treatment, which in most cases is surgical resection [9]. Since a malignant tumor was suspected in this case, proper workup was planned initially but since his symptoms worsened, surgery was performed. During the operation, a large part of the ileum and colon was found to be involved and no definitive cause was identified on the biopsy. However, enlarged lymph nodes may be the cause, which was not identified due to necrosis and gangrene.

\section{Conclusion}

In the present case, a young adult was diagnosed with idiopathic ileocolic intussusception in which a large segment from the ileum to the rectosigmoid junction was involved and no definitive lesion was found by histopathology.

\section{References}

1. Marsicovetere P, Ivatury SJ, White B, Holubar SD. Intestinal Intussusception: Etiology, Diagnosis, and Treatment. Clin Colon Rectal Surg. 2017;30:30-39.

2. Gupta RK, Agrawal CS, Yadav R, Bajracharya A, Sah PL. Intussusceptions in adults: A retrospective interventional series of cases. Healt Renaissance. 2010;8:158-165.

3. Strobel S, Ben-Yakov M, Tessaro MO, Boutis $\mathrm{K}$. Intwosusception: Case report of 2 sisters presenting simultaneously with intussusception. Can Fam Physician. 2017;63:863-865.

4. Hazra NK, Karki OB, Verma M, Rijal D, De A, Nath B. Intussusception in Children: A Short-Term Analysis in a Tertiary Care Hospital. American Journal of Public Health Research. 2015;3:53-56. doi:10.12691/ajphr-3-4A-11

5. Singhal S, Singhal A, Arora PK, Tugnait R, Tiwari B, Malik $\mathrm{P}$, et al. Adult ileo-ileo-caecal intussusception: case report and literature review. Case Rep Surg. 2012;2012:789378.

6. Aberra Y. A case report on post-operative intussusceptions in adult. J Gastrointest Dig Syst. 2017;8:559.

7. Matulich J, Thurston K, Galvan D, Misra S. A case of carcinoid likely causing jejunal intussusception. Case Rep Surg. 2014;2014:949020.

8. Aydin N, Roth A, Misra S. Surgical versus conservative management of adult intussusception: Case series and review. Int J Surg Case Rep. 2016;20:142-146. 
9. Marinis A, Yiallourou A, Samanides L, Dafnios N, Anastasopoulos G, Vassiliou I, et al. Intussusception of the bowel in adults: a review. World J Gastroenterol. 2009; 15:407-411.

10. Kee SK, Kim JO, Kwon OK, Nam SY, Byun KH, Kim KB, et al. A rare ileal intussusception caused by a Lipoma of the ileum. J Korean Surg Soc. 2009;77:59-63.

11. Balamoun H, Doughan S. Ileal lipoma - a rare cause of ileocolic intussusception in adults: Case report and literature review. World J Gastrointest Surg. 2011;3:13-15.

12. Good CA. Tumors of the small intestine. Am J Roentgenol Radium Ther Nucl Med. 1963;89:685-705.

13. Yao T. Primary small intestinal tumors. Stomach Intest. 2001;36:871-881.

14. Boyce S, Khor YP. A colonic submucosal lipoma presenting with recurrent intestinal obstruction attacks. BMJ Case Rep. 2009;2009:bcr.11.2008.1199.

15. Ghidirim G, Mishin I, Gutsu E, Gagauz I, Danch A, Russu S.
Giant submucosal lipoma of the cecum: report of a case and review of literature. Rom J Gastroenterol. 2005;14:393-396.

16. Sinha R. Appendix, colon and rectum. In: Allan PL, Baxter GM, Weston MJ, editors. Clinical Ultrasound. Third Edit. Edinburgh: Churchill Livingstone; 2011. pp. 388-410.

17. Reijnen HA, Joosten HJ, de Boer HH. Diagnosis and treatment of adult intussusception. Am J Surg. 1989;158:25-28.

18. Yalamarthi S, Smith RC. Adult intussusception: case reports and review of literature. Postgrad Med J. 2005;81:174-177.

19. Staff News Brief. Intussusception: Ultrasound for diagnosis and real-time monitoring. Applied Radiology, 27 Dec, 2017. Available from: https://appliedradiology.com/articles/ intussusception-ultrasound-for-diagnosis-and-real-timemonitoring

20. Minaya Bravo AM, Vera Mansilla C, Noguerales Fraguas F, Granell Vicent FJ. Ileocolic intussusception due to giant ileal lipoma: Review of literature and report of a case. Int J Surg Case Rep. 2012;3:382-384. 